\title{
ESTIMACIÓN DEL ALMACENAMIENTO DE CARBONO Y ESTRUCTURA EN BOSQUES CON PRESENCIA DE BAMBÚ (Guadua sarcocarpa) DE LA COMUNIDAD NATIVA BUFEO POZO, UCAYALI, PERÚ.
}

\author{
Diego GARCIA SORIA ${ }^{1}$, Dennis DEL CASTILLO ${ }^{1}$ \\ 1 Instituto de Investigaciones de la Amazonía Peruana, IIAP. Programa en Manejo Integral del Bosque y Servicios \\ Ambientales (PROBOSQUES). Estación Experimental Ucayali. Carretera Federico Basadre km 12.400, Pucallpa, Perú. E- \\ mail:dgarcia@iiap.org.pe.
}

\begin{abstract}
RESUMEN
Se estimó el almacenamiento de carbono (C) de los pacales de la Comunidad Nativa (CC. NN.) Bufeo Pozo en la región Ucayali, al mismo tiempo que se caracterizó su estructura. La zona de estudio presenta una altitud de 280 a 298 m.s.n.m. y una precipitación media anual de 3,000 mm, siendo predominante la especie Paca (Guadua sarcocarpa) en los bosques de colinas bajas. Se estudió la biomasa, necromasa y suelo, utilizando variables de diámetro a la altura del pecho (DAP), altura total (ht), carbono orgánico del suelo (COS), densidad aparente, así como la estructura de estos bosques a través de la abundancia, frecuencia y dominancia absoluta y relativa. Se establecieron 20 parcelas de $10 \times 25 \mathrm{~m}$, agrupadas en cuatro, tomando como mínimo una distancia de $100 \mathrm{~m}$ entre cada parcela y de $500 \mathrm{~m}$ entre cada grupo. El contenido de C total fue de 242.66 toneladas de carbono por hectárea ( $\mathrm{t} \mathrm{C}$ ha ${ }^{-1}$ ), el depósito de $\mathrm{C}$ con mayor contenido es el COS con $120.55 \mathrm{t} \mathrm{C}$ $\mathrm{ha}^{-1}$, seguido de la biomasa y la necromasa con 107.73 y $14.39 \mathrm{t} \mathrm{C}^{-1} \mathrm{r}^{-1}$ respectivamente, la especie con mayor aporte de $\mathrm{C}$ en la biomasa es la Paca con $66.47 \mathrm{t} \mathrm{C} \mathrm{ha}^{-1}$. Esta especie presenta 1660 individuos por ha. Los resultados nos sugieren que los pacales materia de estudio tienen un alto contenido de $\mathrm{C}$, por lo que se aconseja el establecimiento de sistemas de conservación y producción que permitan una prolongada captura del carbono, a través de productos con valor agregado, mitigando de esta forma el cambio climático.
\end{abstract}

PALABRAS CLAVE: Guadua, carbono, biomasa, paca.

\section{CARBON STORAGE AND BAMBU (GUADUA SARCOCARPA) FOREST STRUCTURE ESTIMATION IN THE INDIGENOUS COMMUNITY OF BUFEO POZO, UCAYALI, PERU.}

\begin{abstract}
Carbon $(\mathrm{C}$,) storage was estimated in the pacales of the indigenous community of Bufeo Pozo in the Ucayali region. At the same time the forest structure was characterized. The study area presents an altitude between 280 and 298 m.a.s.l. and annual mean precipitation of $3000 \mathrm{~mm}$. Paca (Guadua sarcocarpa) is the predominant specie in the forest of the low hills. We studied the biomass, necromass and soil using variables of trunk diameter, total height, organic soil carbon, tree density as well as the forest structure through abundance, frequency and absolute and relative dominance. We established 20 plots of $10 \mathrm{~m}$ x $25 \mathrm{~m}$ grouped by four plots with a minimum distance of $100 \mathrm{~m}$ between plots and $500 \mathrm{~m}$ between groups. The total carbon content was estimated in $242.66 \mathrm{tha}^{-1}$. The carbon deposit with highest carbon deposit was the COS with $120.55 \mathrm{tha}^{-1}$ followed by the biomass and necromass with 107.73 and $14.39 \mathrm{tha}^{-1}$ respectively. The specie with highest carbon contribution in biomass is paca with $66.47 \mathrm{t} \mathrm{ha}^{-1}$. Likewise, paca has 1660 individuals per hectare. The results show that the pacales, subject of the study, have a high content of carbon and therefore we recommend to establish conservation and production systems that would allow to keep the carbon sequestered, adding value to the products and contributing to climate change mitigation.
\end{abstract}

KEYWORDS: Guadua, carbon, biomass. 


\section{INTRODUCCIÓN}

A nivel mundial se hacen cada vez más evidentes los efectos del cambio climático, observándose oleadas de frío y de calor cada vez más severas y más frecuentes a lo acostumbrado. Estos cambios se atribuyen principalmente al aumento de las concentraciones de gases de efecto invernadero (GEI) en la atmósfera, tales como el dióxido de carbono $\left(\mathrm{CO}_{2}\right)$, metano $\left(\mathrm{CH}_{4}\right)$, óxido nitroso $\left(\mathrm{N}_{2} \mathrm{O}\right)$, clorofluorocarbonos (CFC) y vapor de agua, que contribuyen al incremento global de la temperatura (Intergovernmental Panel on Climate Change IPCC, 2007). En el Perú, de acuerdo a la segunda comunicación nacional de cambio climático ( S C N C C) de 12010 , e l total de emisiones/remociones de GEI ha sido de 120,023 $\mathrm{Gg}$ de $\mathrm{CO}_{2}$ equivalente $\left(\mathrm{CO}_{2}\right.$ eq $)$ y la principal fuente de estas emisiones es la alteración de bosques atribuida a la deforestación de la Amazonía con el fin de ampliar la frontera agrícola, con un $47.5 \%$ del total de emisiones de $\mathrm{CO}_{2}$. (MINAM, 2010).

Una de las formas de mitigar el cambio climático es la reducción de emisiones de GEI provenientes de la deforestación. El departamento de Ucayali cuenta con 8.7 millones de hectáreas de bosques, que representan aproximadamente el $85 \%$ de su superficie, un aproximado de 10'241,055 ha, de las cuales el $30.61 \%$ son bosques que presentan grandes poblaciones de un tipo de bambú silvestre conocido localmente como "paca" (GOREU, 2008), dando nombre a los "pacales", bosques donde predomina la especie. Aunque la importancia de los bosques para mitigar el cambio climático es reconocida y ampliamente difundida (IPCC, 2007., Angelsen et al., 2009; CIFOR, 2010), las investigaciones realizadas con respecto a la acumulación de carbono en los pacales son inexistentes en la Amazonía peruana, en comparación con otros ecosistemas naturales, además, estos estudios no se han realizado en los ámbitos de los territorios de las comunidades nativas.

En la Comunidad Nativa Bufeo Pozo de la cuenca del Urubamba, Ucayali Perú, existen diferentes usos del suelo, pero generalmente estos usos están destinados a la producción forestal y los cultivos de pan llevar. Entre estos bosques de producción forestal destaca la presencia de los pacales, que aparte de brindar productos maderables, también proveen servicios ambientales como la captura de carbono, protección de suelo y la conservación de la biodiversidad (Camargo et al., 2011). El presente estudio tiene por objetivo contribuir con información específica sobre la acumulación de carbono y la estructura de los pacales. Para ello, se determina el carbono en todos los compartimentos del pacal y se analizan las correlaciones con sus características estructurales.

\section{MATERIALES Y MÉTODOS}

\section{ÁREADELESTUDIO}

El estudio se realizó en los pacales de la Comunidad Nativa Bufeo Pozo, durante los meses de octubre y noviembre del 2012. La comunidad está ubicada en la parte sur del departamento de Ucayali (Figura 1), en las coordenadas $10^{\circ} 54^{\prime} 05^{\prime \prime}$ sur y $73^{\circ} 05^{\prime} 36^{\prime \prime}$ oeste, con altitudes de 280 a 298 m.s.n.m. La precipitación media anual es de $3,000 \mathrm{~mm}$, mostrando una topografía de colinas bajas. La comunidad Bufeo Pozo ha venido realizando aprovechamiento forestal desde hace más de seis años, bajo la modalidad de permiso forestal autorizado por el estado. La paca no tiene un uso conocido por los pobladores de la comunidad.

\section{ESTRUCTURA}

El análisis estructural de los pacales se hace con el propósito de valorar sociológicamente una muestra y establecer su categoría en la asociación. Puede realizarse según las necesidades puramente prácticas de la silvicultura o siguiendo las directrices teóricas de la sociología vegetal. (Otavo et al., 1994) $\mathrm{La}$ estructura horizontal permite evaluar el comportamiento de los árboles individuales y de las especies en la superficie del bosque. Esta estructura puede evaluarse a través de índices que expresan la ocurrencia de las especies, lo mismo que su importancia ecológica dentro del bosque, como es el caso de las abundancias, frecuencias y dominancias, cuya suma relativa genera el índice de valor de importancia (I.V.I.). (Salas et al. 2000)

Para el muestreo de estas variables se usó las mismas parcelas de muestreo del inventario de carbono, evaluándose todos los individuos con DAP $\geq 05 \mathrm{~cm}$. (Figura 2)

\section{ABUNDANCIA.}

Hace referencia al número de individuos por hectárea y por especie en relación con el número total de individuos. Se distingue la abundancia absoluta (número de individuos por especie) y la abundancia relativa (proporción de los individuos de cada especie en el total de los individuos del ecosistema). (Lamprecht, 1990) (Tabla 1)

\section{FRECUENCIA}

Permite determinar el número de parcelas en que aparece una determinada especie, en relación al total de parcelas inventariadas, o existencia o ausencia de una determinada especie en una parcela. La frecuencia absoluta se expresa como un porcentaje $(100 \%=$ existencia de la especie en todas las parcelas), la frecuencia relativa de una especie se determina como su porcentaje en la suma de las frecuencias absolutas de todas las especies. (Melo, 2000). (Tabla 1) 


\section{DOMINANCIA}

Se relaciona con el grado de cobertura de las especies como manifestación del espacio ocupado por ellas y se determina como la suma de las proyecciones horizontales de las copas de los árboles en el suelo. Debido a que la estructura vertical de los bosques naturales tropicales es bastante compleja, la determinación de las proyecciones de las copas de los árboles resulta difícil y a veces imposible de realizar, por esta razón, se utilizan las áreas básales, debido a que existe una correlación lineal alta entre el diámetro de la copa y el fuste. (Lamprecht, 1990)

Bajo este esquema, la dominancia absoluta es la sumatoria de las áreas basales de los individuos de una especie sobre el área especificada y expresada en metros cuadrados y la dominancia relativa es la relación expresada en porcentaje entre la dominancia absoluta de una especie cualquiera y el total de las dominancias absolutas de las especies consideradas en el área de estudio. (Tabla 1)

\section{ÍNDICE DE VALOR DE IMPORTANCIA (I.V.I)}

Formulado por Curtis y Mc Intosh (1951), es posiblemente el más conocido, se calcula para cada especie a partir de la suma de la abundancia relativa, la frecuencia relativa y la dominancia relativa. Permite comparar el peso ecológico de cada especie dentro del bosque. El valor del IVI, similar para diferentes especies registradas en el inventario, sugiere una igualdad o semejanza del bosque en su composición, estructura, calidad de sitio y dinámica. (Braun, 1974) (Tabla 1)

\section{DISEÑO DE LAS PARCELAS DE MUESTREO}

Se establecieron 20 parcelas temporales de $10 \times 25 \mathrm{~m}$ para la evaluación de biomasa, necromasa y carbono orgánico del suelo, agrupadas de cuatro en cuatro, tomando como mínimo una distancia de $100 \mathrm{~m}$ entre cada parcela y de $500 \mathrm{~m}$ entre cada grupo. En cada parcela se utilizó un diseño de muestreo anidado para la evaluación de los diferentes componentes (Figura 2). La biomasa de individuos arbóreos con diámetro a la altura del pecho (DAP) igual o mayor a $10 \mathrm{~cm}$ fue medida en la parcela principal $\left(250 \mathrm{~m}^{2}\right)$, los individuos con DAP de 5 a $9.9 \mathrm{~cm}$ en una parcela de 10 x $10 \mathrm{~m}$, los individuos de 1.5 a $4.9 \mathrm{~cm}$ en una parcela de 5 x $5 \mathrm{~m}$ y los individuos de menos de 1.5 $\mathrm{cm}$ de diámetro se ubicaron dos cuadrantes de $0.5 \mathrm{x}$ $0.5 \mathrm{~m}$ aleatoriamente en la parcela de donde se colectó la biomasa.

La necromasa de los pacales se dividió en árboles muertos en pie, tocones, árboles caídos y hojarasca. Se utilizaron tres métodos de medición: 1) la evaluación en un transecto de $50 \mathrm{~m}$ para madera muerta en el suelo, 2) una parcela de $5 \mathrm{~m}$ x $50 \mathrm{~m}$ para árboles muertos en pie y tocones, en ambos casos se incluyeron individuos con diámetros mayores a $5 \mathrm{~cm}$ y 3) se ubicaron dos cuadrantes de $0.5 \times 0.5 \mathrm{~m}$ aleatoriamente en la parcela de donde se colectó la hojarasca.

Para el muestreo de carbono orgánico del suelo se realizó una evaluación a cinco profundidades, 0-15, $15-30,30-50,50-75$ y $75-100 \mathrm{~cm}$, bajo el protocolo propuesto por (Rügnitz et al., 2009).

\section{BIOMASA AÉREA DE LOS PACALES}

Para el cálculo de la biomasa aérea en los individuos con DAP mayores a $10 \mathrm{~cm}$, se midió a $1.30 \mathrm{~m}$ del suelo utilizando una cinta diamétrica. Para la estimación de la altura de todos los individuos, incluyendo la paca, se usó un clinómetro, tal como aconseja el protocolo estándar de parcelas permanentes de RAINFOR (Phillips y Baker, 2002). Del mismo modo, para el caso de las especies con DAP de 5 a $9.99 \mathrm{~cm}$ y de 1.5 a $4.99 \mathrm{~cm}$, se tomó la altura y el diámetro usando una cinta diamétrica y el clinómetro. Las ecuaciones alométricas usadas fueron las encontradas en la literatura (Tabla 1) y para los valores de densidad de la madera de cada especie identificada se usó la base de datos d i s p o n i b l e e n 1 a $\quad$ W e b (http://hdl.handle.net/10255/dryad.235; Zanne et al., 2009). Para el caso de la Paca se utilizó la ecuación alométrica sugerida por (Recavarren et al., 2011). Así mismo, para el caso de los individuos menores a $1.49 \mathrm{~cm}$ de DAP se instalaron al azar 2 cuadrantes de $0.25 \mathrm{~m}^{2}$ de donde se colectó la totalidad de la biomasa tomando su peso fresco total (Figura 2). Posteriormente, se tomó una muestra para llevar al laboratorio y secarla a $65{ }^{\circ} \mathrm{C}$ hasta obtener un peso seco constante, se consideró la fracción de carbono de 0.5 del peso seco obtenido (IPCC, 2003).

\section{CARBONO DE NECROMASA}

Para medir los árboles caídos se usó el método de intersección de línea en el cual se estableció un transecto de $50 \mathrm{~m}$, ubicado en uno de los lados de la parcela (Rügnitz et al., 2009). (Figura 2): En ese transecto se midieron los diámetros de los troncos que se interceptan por la línea del transecto, y en el caso de troncos de forma elíptica se tomaron dos diámetros, conjuntamente con la medición del diámetro se clasificó los troncos en tres categorías según su estado de pudrición. (Tabla 2)

Posteriormente, para obtener el contenido total de necromasa de las tres categorías, se multiplica el resultado de cada categoría por 0.5 para la categoría de árboles sólidos, por 0.3 para la categoría de árboles intermedios y por 0.15 para la categoría de árboles podridos.

Para el caso de los árboles muertos en pie y tocones se usó la misma metodología, midiendo su altura y diámetro, usando el clinómetro y/o regla medidora y la cinta diamétrica respectivamente. (Tabla 2)

Para el caso de la necromasa herbácea se instalaron 
al azar 2 cuadrantes de $0.25 \mathrm{~m}^{2}$, de donde se colectó la totalidad de la necromasa, tomando su peso fresco total, posteriormente se tomó una muestra para llevar al laboratorio en donde se secó a $65^{\circ} \mathrm{C}$ hasta obtener una peso seco constante, se consideró la fracción de carbono de 0.5 del peso seco obtenido (IPCC, 2003). (Figura 2).

\section{CARBONO ORGÁNICO DEL SUELO (COS)}

Para el análisis del carbono orgánico del suelo se consideraron 5 profundidades de muestreo: de $0-15$, $15-30,30-50,50-75$ y $75-100 \mathrm{~cm}$. La metodología de cuantificación se basó en la densidad aparente medida por el método del cilindro y el análisis químico de la fracción de carbono se realizó en el laboratorio de la Universidad Agraria de la Molina, utilizando el método de ácido crómico (Walkley y Black, 1934).

\section{ARREGLOS ESTADÍSTICOS}

Para el caso de los pacales $(n=20)$ se realizó una comparación de medias mediante la prueba de Kruskal y Wallis (1952) con un nivel de confianza de $95 \%($ alfa $=0.05)$, utilizando el software SPSS.

\section{RESULTADOS Y DISCUSIÓN}

\section{ESTRUCTURA}

Los valores en DAP variaron de $5.09 \mathrm{~cm}$ a $67.06 \mathrm{~cm}$, con una media de $10.03 \mathrm{~cm}$; en un total de 2,141 individuos medidos. Las especies con altos IVI presentes en las 20 parcelas fueron: Paca (Guadua sarcocarpa), Tahuari (Tabebuia serratifolia), Requia (Guarea guidonia), Shimbillo (Inga sertulifera DC), Quillobordon (Aspidosperma vargasii A.DC), Ubilla (Coussapoa sp), Cumala (Virola elliptica A.C. Sm), Carahuasca (Guatteria pteropus Benth), Caimitillo (Pouteria guianensis Aubl.) y Cetico (Cecropia membranácea); los valores de frecuencia y densidad relativas elevados determinaron el IVI, estas especies se consideran de importancia estructural. (Tabla 3).

En todas las parcelas se constata la presencia de la paca, estando presente en un total de 20 parcelas, la Requia (Guarea guidonia) está presente en 14, el Shimbillo (Inga sertulifera DC) en 12, el Quillobordon (Aspidosperma vargasii A.DC) y la Ubilla (Coussapoa sp) en 10, la Carahuasca (Guatteria pteropus Benth), la Cumala (Virola elliptica A.C. Sm) y el Tahuari (Tabebuia serratifolia) en 9, siendo la presencia del resto de especies inferior a nueve individuos por parcela. (Tabla 3)

\section{CARBONO}

El carbono orgánico del suelo (COS) presente en la zona de estudio alcanzó los niveles más elevados de C en relación a los depósitos analizados, con 120.53 $\pm 8.43 \mathrm{t} \mathrm{C} \mathrm{ha}^{-1}$. Los niveles obtenidos al medir la biomasa aérea fueron significativamente similares en su contenido total de $\mathrm{C}$, con $107.73 \pm 17.59 \mathrm{tC} \mathrm{ha}^{-1}$ (Figura 3). Asimismo, en la zona de estudio, la necromasa fue el depósito que contenía menos carbono total, con $14.39 \pm 3.84 \mathrm{t} \mathrm{C} \mathrm{ha}^{-1}$. Resalta el hecho de que la Paca es la especie que aporta más $\mathrm{C}$ a la biomasa aérea con un total de $66.46 \pm 12.34 \mathrm{tC} \mathrm{ha}^{-1}$ (Figura 3).

\section{CARBONOEN LA BIOMASA AÉRA}

El contenido de $C$ en la biomasa aérea de los pacales encontrados en este estudio $107.73 \pm 17.59 \mathrm{t} \mathrm{C} \mathrm{ha}^{-1}$, se asemeja a los niveles para biomasa aérea de pacales naturales descritos por (Camargo et al., 2007), en la Vereda Calle larga, finca Nápoles en Montenegro, Colombia, donde reportó $126.00 \pm$ $41.7 \mathrm{t} \mathrm{C} \mathrm{ha}^{-1}$, en condiciones climáticas y de altura bastante similares. Sin embargo, la cantidad de culmos de paca encontrados difieren en 1,660 en este estudio y en 4,050 culmos ha $^{-1}$ reportados por Camargo et al., 2007.

La explicación de estas variaciones puede ser atribuida a la clasificación hecha por Camargo de los bosques como "bosques naturales de bambú", lo que difiere del presente estudio y de la clasificación realizada de los bosques como "bosques mixtos con presencia de bambú". Los datos reportados coinciden con los trabajos realizados por (Sharma y Vu Tan Phuong, 2013) en Vietnam, donde reportan un total de $107.00 \mathrm{tC} \mathrm{ha}^{-1}$, cifra prácticamente igual a la encontrada en este estudio y que nos hacen deducir que las zonas estudiadas tienen la misma estructura y categoría.

\section{CARBONO EN LA NECROMASA}

Abordando el tema del C en la necromasa, se encontró un promedio de $14.39 \pm 3.84 \mathrm{t} \mathrm{C}^{-1}$, valor mucho mayor a los reportados por Isagi et al. (1997) de $3.8 \mathrm{t} \mathrm{C} \mathrm{ha}^{-1}$ en los bambusales de Japón. Esta disminución de los valores obedece a que el estudio de Isagi et al se basa en bambusales donde predomina la especie Phyllostachys pubescens, especie que no tiene una arquitectura y fisiología ni tan siquiera parecida a la Guadua sarcocarpa, especie predominante en este estudio.

\section{CARBONO ORGÁNICO DEL SUELO (COS)}

El contenido de COS en la zona de estudio presenta $120.53 \pm 8.43 \mathrm{t} \mathrm{C}^{-1} \mathrm{Ca}^{-1}$, constituyéndose como el depósito de carbono más importante en este tipo de bosques. Estos datos difieren un poco a los encontrados por Isagi et al. (1997), quien reportó un almacenamiento de $94.2 \mathrm{t} \mathrm{C} \mathrm{ha}^{-1}$, explicándose esta variación por el aporte de la especie Phyllostachys pubescens al suelo, especie que no tiene una fisiología parecida a la paca. 


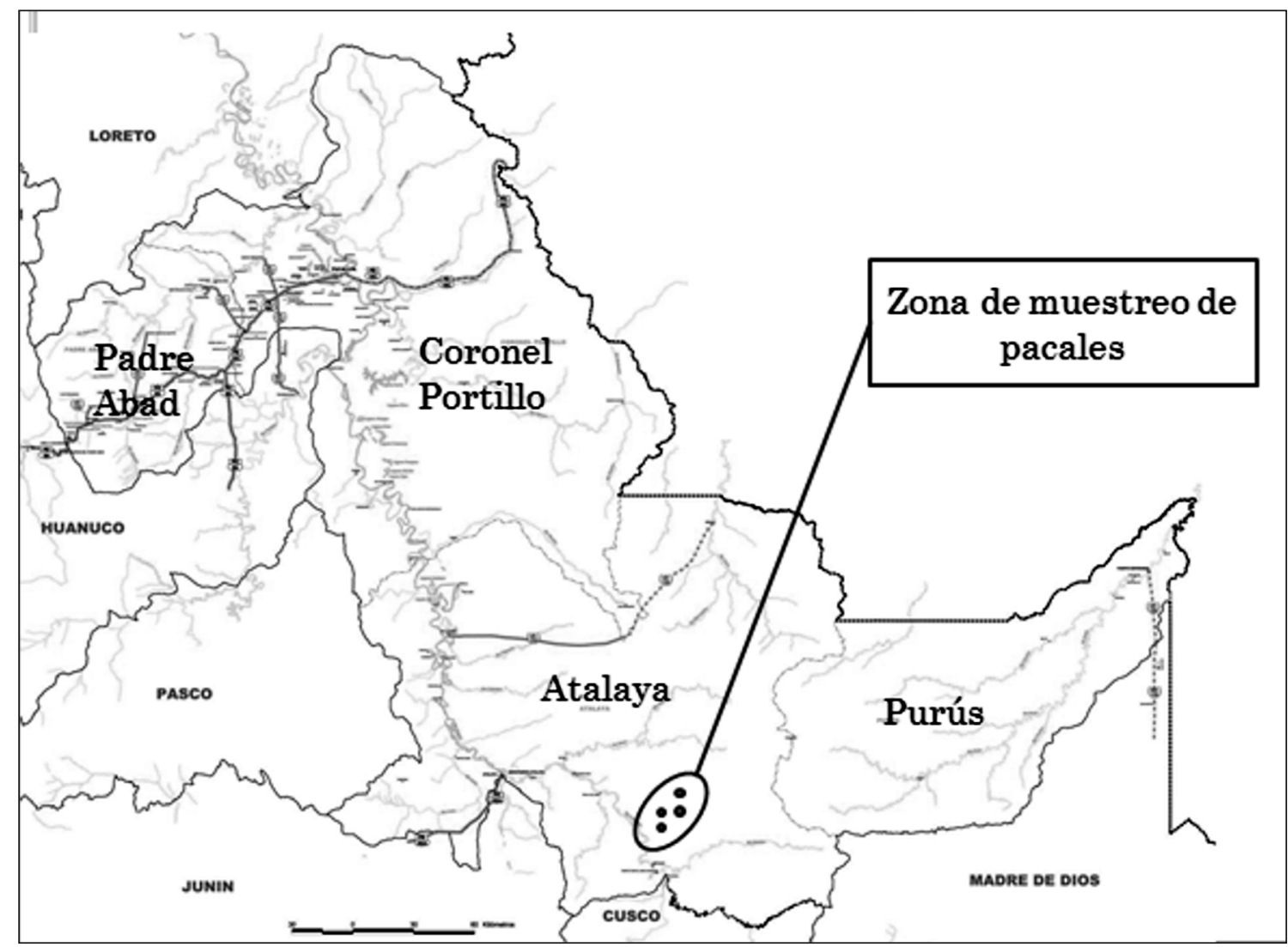

Figura 1. Ubicación de los pacales en la CC. NN. Bufeo Pozo.

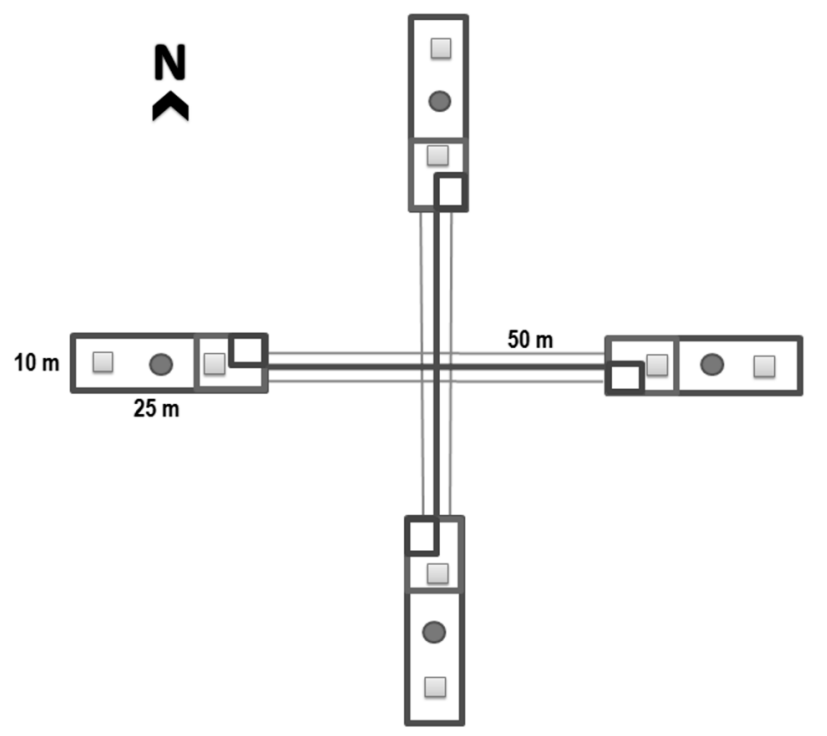

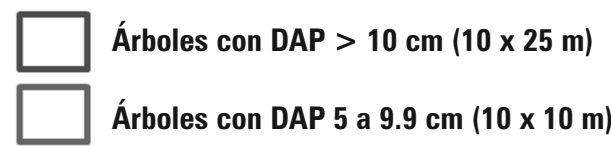

$\square$ Árboles con DAP 1.5 a $4.9 \mathrm{~cm}(5 \times 5 \mathrm{~m})$

Árboles caidos $50 \mathrm{~m}$ lineales

Árboles muertos en pie y tocones (5 x 50 m)

Hojarasca y herbáceas $(0.5 \times 0.5 \mathrm{~m})$

$\operatorname{COS}$ a 5 profundidades $(1 \mathrm{~m})$

$\overline{\text { Figura 1. Diseño anidado de la parcela }}$ de muestreo de estructura, biomasa, necromasa y de carbono en el suelo. Las medidas de la parcela principal y de las parcelas anidadas se indican en la figura. 


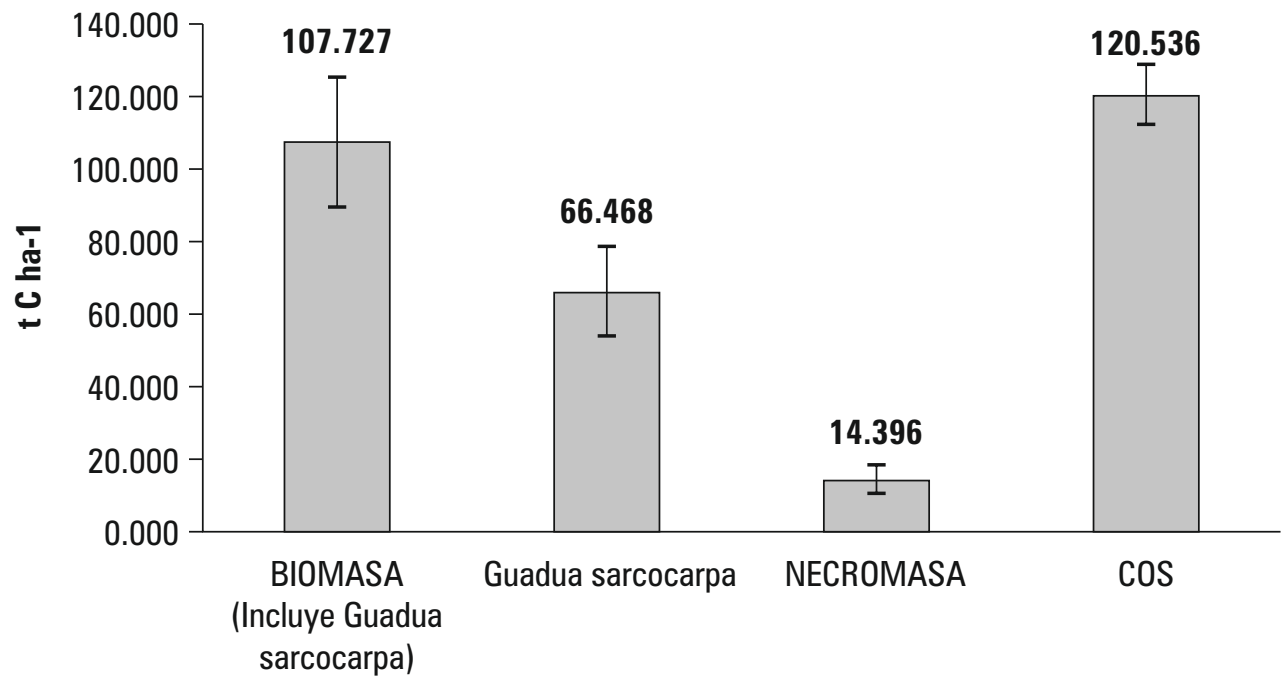

\section{Depositos}

Figura 1. Almacenamiento de carbono total en diferentes depósitos en los pacales de la CC.NN. Bufeo Pozo. Las barras indican el error estándar.

Tabla 1. Fórmulas para el cálculo de variables estructurales en los pacales de la CC.NN. Bufeo Pozo.

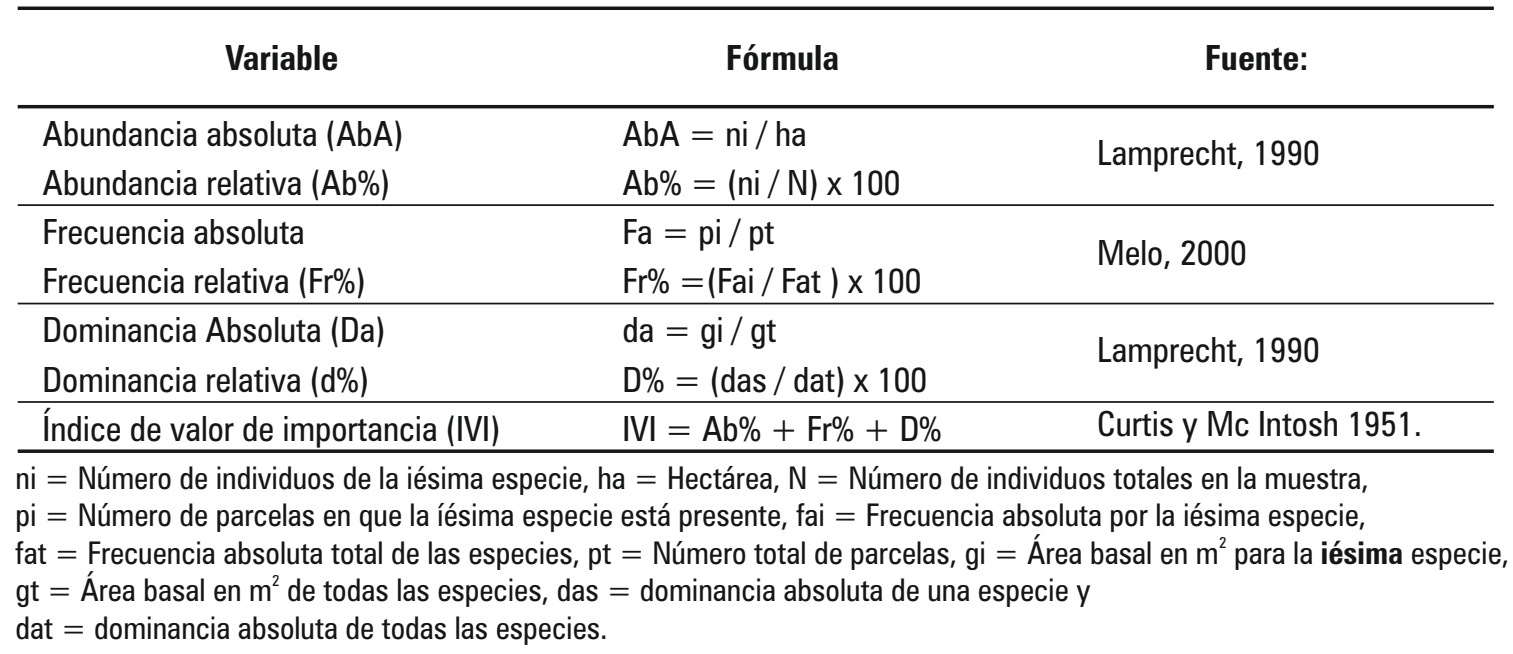


Tabla 1. Ecuaciones alométricas para la estimación de biomasa en la paca, otros árboles presentes y necromasa presentes en los pacales de la CC. NN. Bufeo Pozo.

\begin{tabular}{|c|c|c|}
\hline Especificación: & Ecuación: & Fuente: \\
\hline $\begin{array}{c}\text { Árboles y otras } \\
\text { palmeras }>10 \mathrm{~cm} \\
\text { de DAP. }\end{array}$ & $B=\exp \left(-2.977+\ln \left(p D 2^{*} H\right)\right)$ & (Chave et al., 2005) \\
\hline $\begin{array}{c}\text { Árboles y otras } \\
\text { palmeras de } 5 \text { a } 9.9 \mathrm{~cm} \\
\text { de DAP. }\end{array}$ & $B=13.312 * \ln (D A P)-20.237$ & (Chave et al., 2005) \\
\hline $\begin{array}{c}\text { Árboles y arbustos } \\
\text { de } 1.5 \text { a } 4.9 \mathrm{~cm} \\
\text { de DAP. }\end{array}$ & $B=\exp (-1.7689+2.377 * \ln (\mathrm{DAP}))$ & (Nascimento y Laurence, 2002) \\
\hline Para la paca & $B=7.2581 * D-7.0782$ & (Recavarren et al., 2011) \\
\hline $\begin{array}{l}\text { Biomasa } \\
\text { herbácea }\end{array}$ & $B=10000 / \mathrm{Ap}^{*}\left(\mathrm{Bh}{ }^{*} \mathrm{Bsm} / \mathrm{Bhm}\right) / 1000$ & IPCC 2003 \\
\hline Árboles caídos & $V=\mathrm{n} 2+\frac{\left(\mathrm{D}_{1}{ }^{2}+\mathrm{D}_{2}{ }^{2}+\ldots+\mathrm{D}_{\mathrm{n}}{ }^{2}\right)}{8^{*} \mathrm{~L}} \cdot \mathrm{Cp}$ & IPCC 2003 \\
\hline $\begin{array}{c}\text { Árboles } \\
\text { muertos en pie } \\
\text { y tocones }\end{array}$ & $\mathrm{B}=\pi^{*}((\mathrm{D} / 2) 2 * \mathrm{H}) * 0.3 / 1000$ & IPCC 2003 \\
\hline $\begin{array}{c}\text { Necromasa } \\
\text { herbácea }\end{array}$ & $B=10000 / \mathrm{Ap}^{*}\left(\mathrm{Nh}{ }^{*} \mathrm{Nsm} / \mathrm{Nhm}\right) / 1000$ & IPCC 2003 \\
\hline
\end{tabular}

B: biomasa (kg); Ln: Logaritmo base 10; exp: exponencial; p: densidad básica de la especie; DAP: diámetro $(\mathrm{cm})$ del tronco a la altura del pecho $(1,3 \mathrm{~m})$; $\mathrm{Ht}$ : altura total $(\mathrm{m})$; V: volumen; $\mathrm{D}$ : diámetro de pieza; L: longitud de transecto $(\mathrm{m}) \mathrm{C}$ : carbono en biomasa; Cp: Categoría de pudrición (0.5, 0.3 y 0.15); Ap: Área de la parcela; Bh: Biomasa húmeda; Bsm: Biomasa seca de la muestra; Bhm: Biomasa húmeda de la muestra; Nh: Necromasa húmeda; Nsm: Necromasa seca de la muestra; Nhm: Necromasa húmeda de la muestra Fc: fracción de C $(0,5)$ 
FOLIA

\begin{tabular}{|c|c|c|c|c|c|c|c|c|}
\hline ID & Especie & AbA/ha & $\mathbf{A b} \%$ & $\mathrm{Fa}$ & $\mathrm{Fr} \%$ & $\mathrm{Da} / \mathrm{ha}$ & D\% & IVI \\
\hline 1 & Paca & 1660 & 77.53 & 1.00 & 9.48 & 0.20 & 19.96 & 106.97 \\
\hline 2 & Tahuari & 16 & 0.75 & 0.45 & 4.27 & 0.09 & 8.80 & 13.81 \\
\hline 3 & Requia & 43 & 1.98 & 0.70 & 6.64 & 0.05 & 4.97 & 13.59 \\
\hline 4 & Shimbillo & 32 & 1.47 & 0.60 & 5.69 & 0.06 & 6.23 & 13.39 \\
\hline 5 & Quillobordon & 26 & 1.19 & 0.50 & 4.74 & 0.07 & 6.77 & 12.70 \\
\hline 6 & Ubilla & 13 & 0.59 & 0.50 & 4.74 & 0.05 & 5.33 & 10.66 \\
\hline 7 & Cumala & 18 & 0.84 & 0.45 & 4.27 & 0.05 & 4.99 & 10.10 \\
\hline 8 & Carahuasca & 14 & 0.65 & 0.45 & 4.27 & 0.04 & 4.01 & 8.93 \\
\hline 9 & Caimitillo & 12 & 0.54 & 0.30 & 2.84 & 0.04 & 3.86 & 7.24 \\
\hline 10 & Cetico & 14 & 0.65 & 0.25 & 2.37 & 0.04 & 4.16 & 7.18 \\
\hline 11 & Chimicua & 15 & 0.70 & 0.40 & 3.79 & 0.02 & 1.54 & 6.04 \\
\hline 12 & Quinilla & 10 & 0.47 & 0.25 & 2.37 & 0.03 & 2.75 & 5.59 \\
\hline 13 & Copaiba & 5 & 0.21 & 0.15 & 1.42 & 0.04 & 3.77 & 5.40 \\
\hline 14 & Huqui huqui & 17 & 0.77 & 0.30 & 2.84 & 0.01 & 1.37 & 4.98 \\
\hline 15 & Mashonaste & 12 & 0.54 & 0.25 & 2.37 & 0.01 & 1.24 & 4.15 \\
\hline 16 & Moena & 12 & 0.56 & 0.20 & 1.90 & 0.02 & 1.57 & 4.03 \\
\hline 17 & Caupuri & 12 & 0.56 & 0.25 & 2.37 & 0.01 & 0.51 & 3.44 \\
\hline 18 & Tanque & 11 & 0.49 & 0.25 & 2.37 & 0.01 & 0.53 & 3.39 \\
\hline 19 & Palta moena & 10 & 0.44 & 0.20 & 1.90 & 0.01 & 0.63 & 2.97 \\
\hline 20 & Quina quina & 13 & 0.61 & 0.15 & 1.42 & 0.01 & 0.93 & 2.96 \\
\hline \multicolumn{2}{|c|}{ Sub total 20 sp importantes } & 1960 & 91.55 & 7.60 & 72.04 & 0.84 & 83.93 & 247.51 \\
\hline \multicolumn{2}{|c|}{ Sub total $37 \mathrm{sp}$ restantes } & 181 & 8.45 & 2.95 & 27.96 & 0.16 & 16.07 & 52.49 \\
\hline \multicolumn{2}{|c|}{ TOTAL } & 2141 & 100.00 & 10.55 & 100.00 & 1.00 & 100.00 & 300.00 \\
\hline
\end{tabular}




\section{BIBLIOGRAFÍA CITADA}

Angelsen, A., Con Brockhaus, M., Kanninen, M., Sills, E; Sunderlin, WD., Wertz-Kanounnikoff, S., 2009. La implementación de REDD+: estrategia nacional y opciones de política. Bogor, Indonesia CIFOR. 362 p.

Braun, J. 1974. Fitosociología. Bases para el estudio de las comunidades vegetales. H. Blume Ediciones. Madrid. $820 \mathrm{P}$.

CIFOR (Center for International Forestry Research). 2010. Simply REDD, CIFOR's Guide for Forest, Climate Change and REDD. Guide Book, CIFOR, Bogor, Indonesia. 16 p.

Camargo, J.C., Chará, J., Giraldo, L., Chará-Serna, A., Pedraza, G., 2011. Beneficios de los corredores ribereños de Guadua angustifolia en la protección de ambientes acuáticos en la Ecorregión Cafetera de Colombia. 1. Efectos sobre propiedades del suelo. Recursos Naturales y Ambiente 61: 47-53.

Chave, J., Andalo, A., Brown, S., Cairns, M.A., Chambers, J.Q., Eamus, D., Fölster, H.,Fromard, F., Higuchi, N., Kira, T., Lescure, J.-P., Nelson, B.W., Ogawa, H., Puig, H.,Rie'ra, B., Yamakura, T., 2005. Tree allometry and improved estimation of carbonstocks and balance in tropical forests. Oceologia 145, $87-99 \mathrm{p}$.

GOREU. 2008. Plan vial departamental participativo de Ucayali 2009 - 2018, Ministerio de Transportes y Telecomunicaciones. Pucallpa. $137 \mathrm{p}$.

Isagi, Y., Kawahara, T., Kamo, K., Ito, H. 1997. Net production and carbon cycling in a bamboo Phyllostachys pubescens stand. Plant Ecology 130: $41-52 \mathrm{p}$.

IPCC (Intergovernmental Panel on Climate Change). 2007. Synthesis report: Climate change 2007. Ginebra, Suiza. 52 p.

IPCC (Intergovernmental Panel on Climate Change), 2003. Orientación sobre las buenas prácticas para el uso de la tierra, cambio de uso de la tierra y silvicultura (UTCUTS). Ginebra, Suiza. $628 \mathrm{p}$

Kruskal, W.H., Wallis, W.A., 1952. Use of ranks on one-criterion variance analysis. Journal of the American Statistical Association, 47: 583-621 p.

Lamprecht, H. 1990. Silvicultura en los trópicos: Los ecosistemas forestales en los bosques tropicales y sus especies arbóreas posibilidades y métodos para un aprovechamiento sostenido - . Trad. A Carrillo. Eschborn, República Federal de Alemania, GTZ. 335 p.

Melo, O. 2000. Evaluación ecológica y silvicultural de los fragmentos de vegetación secundaria, ubicados en áreas de bosque seco tropical en el norte del departamento del Tolima. Universidad del Tolima. Facultad de Ing. Forestal. Ibagué. $150 \mathrm{p}$.

MINAM, 2010. Segunda Comunicación Nacional del Perú a la Convención Marco de las Naciones Unidas sobre Cambio Climático (SCNCC), Primera edición. Lima, Perú. 208 p.

Nascimento, H. E. M., Laurence, W. F., 2002. Total Aboveground biomass in central Amazonian rainforest: a landscape.sclae study. Forest Ecol. Manage. 168, 311-321 p.

Otavo, E. 1994. Análisis estructural de la vegetación. En: Sánchez, H. y Castaño, C.: Aproximación a la definición de criterios para la zonificación y el ordenamiento forestal en Colombia. Ministerio del Medio Ambiente. OIMT. PNUD. 72-81 p.

Phillips, O.; Baker, T. 2002. Manual de campo para la remedición y establecimiento de parcelas. Rainfor. Proyecto PAN-AMAZONIA. $18 \mathrm{p}$.

Salas, G.; Melo, O. 2000. Estructura, biodiversidad y dinámica sucesional en los ecosistemas húmedos tropicales del pacifico colombiano. En: Seminario Internacional De Ecología. El funcionamiento de los ecosistemas tropicales. Fundación Universitaria Manuela Beltrán. Santa fe de Bogotá. $75-85$ p.

Recavarren, P; Delgado M.; Angulo, M.; León, A.; Castro, A. 2011. Proyecto REDD en Áreas Naturales Protegidas de Madre de Dios. Insumos para la elaboración de la línea base de carbono. Asociación para la Investigación y el Desarrollo Integral-AIDER. Lima, Perú. 201 p.

Rügnitz, M. T.; Chacón, M. L.; Porro, R. 2009. Guía para la Determinación de Carbono en Pequeñas Propiedades Rurales - 1. ed. - Lima, Perú.: Centro Mundial Agroflorestal (ICRAF) / Consórcio Iniciativa Amazónica (IA). 79 p.

Sharma, B.D.; Vu Tan Phuong, S.R. S. 2013. Generating Forest Biomass Carbon Stock Estimates for Mapping the Potential of REDD+ to deliver biodiversity conservation in Vietnam. SNV - The Netherlands Development Organisation, Ho Chi Minh City. 20 p.

Walkley, A.; Black, AI. 1934. An examination of the Degtjoreff method for determination soil organic matter, and a proposed codification of the cromic acid titration method. Soil Science 37:29-38 p.

Zanne, A. E.; López-González, G.; Coomes, D. A.; Ilic, J.; Jansen, S.; Lewis, S. L.; Miller, R. B.; Swenson, N. G.; Wiemann, M. C.; Chave, J. 2009. Global Wood density database. Dryad. Identifier.

(http://hdl.handle.net/10255/dryad.235.). Acceso: $15 / 11 / 2012$. 
